Bull. Austral. Math. Soc.

VOL. $70(2004) \quad[87-100]$

\title{
CHARACTERISATION OF THE SUB-RIEMANNIAN ISOMETRY GROUPS OF $H$-TYPE GROUPS
}

\author{
Kang-Hai TAN and XiaO-Ping Yang
}

\begin{abstract}
For a $H$-type group $G$, we first give explicit equations for its shortest sub-Riemannian geodesics. We use properties of sub-Riemannian geodesics in $G$ to characterise the isometry group $I S O(G)$ with respect to the Carnot-Carathéodory metric. It turns out that $I S O(G)$ coincides with the isometry group with respect to the standard Riemannian metric of $G$.
\end{abstract}

\section{INTRODUCTION}

For a $H$-type group $G$, the aim of this paper is to study in detail some properties of its shortest sub-Riemannian geodesics and to give a full characterisation of the isometry group with respect to the Carnot-Carathéodory metric.

The Lie groups of $H$-type are first introduced by Kaplan in [11]. Let $G$ be a Carnot group (see [7]) of step 2 . That is, $G$ is a simply connected Lie group whose Lie algebra $\mathcal{G}$ admits a nilpotent stratification of step 2: $\mathcal{G}=V_{1} \oplus V_{2}$, and $\left[V_{1}, V_{1}\right]=V_{2}$, whereas $\left[V_{1}, V_{2}\right]=0$. From the definition, the centre of $G$ is $\exp \left(V_{2}\right)$ where $\exp$ is the exponential map which is a global diffeomorphism. We assume that a left-invariant Riemannian metric $\langle\cdot, \cdot\rangle$ is given on $G$ for which $V_{1}, V_{2}$ are mutually orthogonal. We denote by $H$-type groups the subbundle spanned by the system of left-invariant vector fields $\left\{X_{1}, \ldots, X_{m_{1}}\right\}$ such that $\left\{X_{1}, \ldots, X_{m_{1}}\right\}$ is an orthonormal basis of $V_{1}$ where $m_{1}=\operatorname{dim}\left(V_{1}\right)$. From the stratification condition and the Chow connectivity theorem $([6])$, the structure of $(H$-type groups, $\langle\cdot, \cdot\rangle)$ induces the so-called Carnot-Carathéodory metric $d_{c}$ : for any $p, q \in G$,

$$
d_{c}(p, q)=\inf _{\gamma}\left\{\int_{a}^{b}|\dot{\gamma}(s)| d s\right\}
$$

where the infimum is taken over all horizontal curves $\gamma$ connecting $p$ to $q$, that is, all absolutely continuous curves joining $p$ and $q$ whose derivatives are in $H$-type groups almost everywhere. $d_{c}$ is left-invariant, that is, $d_{c}\left(p_{0} p, p_{0} q\right)=d_{c}(p, q)$ for any $p_{0}, p, q \in G$, and is 1-homogeneous with respect to the natural dilations, that is $d_{c}\left(\delta_{s} p, \delta_{s} q\right)=s d_{c}(p, q)$

Received 12th January, 2004

The authors thank the referee for useful comments and suggestions.

Copyright Clearance Centre, Inc. Serial-fee code: 0004-9727/04 \$A2.00+0.00. 
for any $s>0, p, q \in G$, where $\delta_{s} p=\exp \left(s \xi_{1}+s^{2} \xi_{2}\right)$ for $p=\exp \left(\xi_{1}+\xi_{2}\right), \xi_{i} \in V_{i}$. A horizontal curve is called a sub-Riemannian geodesic if it locally realises the CarnotCarathéodory distance. We call $G$ is a $H$-type group if $G$ is a Carnot group of step 2 and moreover its Lie algebra $\mathcal{G}$ satisfies the following statement: for every $\eta \in V_{2}$, such that $|\eta|=1$, the map $J(\eta): V_{1} \rightarrow V_{1}$ defined by

$$
\left\langle J(\eta) \xi^{\prime}, \xi^{\prime \prime}\right\rangle=\left\langle\left[\xi^{\prime}, \xi^{\prime \prime}\right], \eta\right\rangle, \quad \eta \in V_{2}, \xi^{\prime}, \xi^{\prime \prime} \in V_{1}
$$

is orthogonal. The simplest $H$-type group is the Heisenberg group $\mathbb{H}^{n}$ (see [23]) which is, by definition, simply $\mathbb{R}^{2 n+1}$, with the noncommutative group law

$$
p p^{\prime}=(x, y, t)\left(x^{\prime}, y^{\prime}, t^{\prime}\right)=\left(x+x^{\prime}, y+y^{\prime}, t+t^{\prime}+\frac{1}{2}\left(\left\langle x^{\prime}, y\right\rangle-\left\langle x, y^{\prime}\right\rangle\right)\right)
$$

where we have let $x, x^{\prime}, y, y^{\prime} \in \mathbb{R}^{n}, t, t^{\prime} \in \mathbb{R}$. A simple computation shows that the leftinvariant vector fields

$$
X_{j}(p)=\frac{\partial}{\partial x_{j}}+\frac{y_{j}}{2} \frac{\partial}{\partial t}, X_{n+j}(p)=\frac{\partial}{\partial y_{j}}-\frac{x_{j}}{2} \frac{\partial}{\partial t}, j=1, \ldots, n,
$$

and $T=\frac{\partial}{\partial t}$ span the Lie algebra $\left(\mathbb{R}^{2 n+1}\right)$ of $\mathbb{H}^{n}$. Moreover $\left[X_{j}, X_{n+k}\right]=-T \delta_{j k}, j$, $k=1, \ldots, n$, and all other commutators are trivial. Note that for the Heisenberg group $\mathbb{H}^{n}$ which is endowed with a Riemannian metric $\langle\cdot, \cdot\rangle$ such that $\left\{X_{1}, \ldots, X_{2 n+1}, T\right\}$ is an orthonormal basis, the map $J$ defined by (1.1) can be explicitly written:

$$
\begin{aligned}
J(T) X_{i} & =-X_{n+i}, \\
J(T) X_{n+i} & =X_{i}
\end{aligned}
$$

for $i=1, \ldots, n$.

$H$-type groups appear naturally in the Iwasawa decomposition of semisimple Lie groups of real rank one. Since they were introduced in [11] by Kaplan, many authors have contributed to analysis and geometry on these groups, see $[5,12,13,14,15,16$, 20]. In [12] Kaplan studied the Riemannian geodesics and characterised the isometry group with respect to the Riemannian metric $\langle\cdot, \cdot\rangle$. In [13, p. 33-p. 35] Korányi gave an explicit description of sub-Riemannian geodesics. What we are interested in is how to characterise shortest sub-Riemannian geodesics. In fact, in analysis on $H$-type groups the most useful information for sub-Riemannian geodesics is the explicit equations for shortest sub-Riemannian geodesics, see $[\mathbf{2}, 3,20,24]$ for applications in the Heisenberg group. In the case of the Heisenberg group, [20] listed without proof the explicit equations for the shortest sub-Riemannian geodesics, and $[2,3]$ independently gave proofs. But the proofs of $[2,3]$ are not trivial. In this paper, we present a direct and simpler proof even for $H$-type groups and explicitly give equations of shortest sub-Riemannian geodesics (see Theorem 2.3). 
Next we shall use properties of sub-Riemannian geodesics to give a full characterisation of the isometry group of sub-Riemannian isometries. A sub-Riemannian isometry of $G$ is a map $f: G \rightarrow G$ such that $d_{c}(f(p), f(q))=d_{c}(p, q)$ for any $p, q \in G$. Note that in this case, since $d_{c}$ is not smooth (see for example $[9,4,2]$ ), we cannot use the method in [12]. We shall prove that the sub-Riemannian isometry group coincides with the isometry group for the standard Riemannian metric $\langle\cdot, \cdot\rangle$ (see Theorem 3.4 and Theorem 3.5). Our proof essentially depends on two facts on shortest sub-Riemannian geodesics (see Corollary 2.5). One is that a geodesic is globally shortest if and only if it is a ray. The other is that there are infinitely many shortest geodesics connecting two given points $p, q$ if and only if $p^{-1} \cdot q$ is in the centre of $G$.

Notations. The letter $G$ will always represent a $H$-type group. We use $p, q, p^{\prime}, q^{\prime}, p_{0}$, $q_{0}, \ldots$ to denote elements in $G$; adopt $\xi, \xi^{\prime}, \xi^{0}, W, W^{0}, \ldots$ to denote elements in $\mathcal{G}$ and $\xi_{1}, \xi_{1}^{\prime}, \xi_{1}^{0}$ elements in $V_{1}$ while $\xi_{2}, \xi_{2}^{\prime}, \xi_{2}^{0}$ in $V_{2}$. We shall write $p=\left(\xi_{1}(p), \xi_{2}(p)\right)$ or $p=\left(\xi_{1}, \xi_{2}\right)$ when no confusion will be caused. The unit element of $G$ is denoted by 0 . Let $G^{*}:=G \backslash \exp \left(V_{2}\right)$ be the set of all elements of the form $p=\left(\xi_{1}(p), 0\right)$. If $p \in G^{*}$ we shall sometimes use $s p$ to denote $\delta_{s} p$.

\section{Properties of SUB-RiemanNiAN GeOdesics}

This section is devoted to studying some properties of sub-Riemannian geodesics in $H$-type groups. The equations of sub-Riemannian geodesics can be easily deduced from the Maximum Principle of Optimal Control Theory. That is, every sub-Riemannian geodesic must satisfy a Hamiltonian equation determined by the horizontal bundle $H$-type groups. It is clear that every sub-Riemannian geodesic is smooth (see for example[19]). In [13] Korányi also found the equations of sub-Riemannian geodesics by minimising the arc length functional among the curve family of horizontal curves joining two given points. The two methods are equivalent. The existence of shortest sub-Riemannian geodesics can be easily inferred from [8, Theorem 1.10]. What we are concerned with is the uniqueness of shortest sub-Riemannian geodesics. For more on the theory of subRiemannian geodesics in general sub-Riemannian manifolds we refer to the book [21].

Our theorem is based on the following statement developed by Korányi in [13].

Proposition 2.1. (Equations of sub-Riemannian geodesics.) Given a point $p_{0}=\left(\xi_{1}^{0}, \xi_{2}^{0}\right)\left(p_{0} \neq 0\right)$ in $G$, the equations of sub-Riemannian geodesics $\gamma(s)$ $=\left(\xi_{1}(s), \xi_{2}(s)\right), s \in[0,1]$ connecting 0 to $p_{0}$ are:

(1) If $\xi_{2}^{0}=0$, then

$$
\xi_{1}(s)=s \xi_{1}^{0}, \quad \xi_{2}(s)=0
$$

(2) If $\xi_{2}^{0} \neq 0$, then, with the notation $T_{0}^{\prime}=\xi_{2}^{0} /\left|\xi_{2}^{0}\right|$, 


$$
\begin{aligned}
& \xi_{1}(s)=(\cos (s \tau)-1) W_{0}+\sin (s \tau)\left(J\left(T_{0}^{\prime}\right) W_{0}\right) \\
& \xi_{2}(s)=\frac{1}{2}(s \tau-\sin (s \tau))\left|W_{0}\right|^{2} T_{0}^{\prime}
\end{aligned}
$$

where $\tau$ is a positive solution of

$$
\frac{1-\cos \tau}{\tau-\sin \tau}=\frac{\left|\xi_{1}^{0}\right|^{2}}{4\left|\xi_{2}^{0}\right|}
$$

and $W_{0}$ is determined in the case $\xi_{1}^{0} \neq 0$ by

$$
\xi_{1}^{0}=(\cos \tau-1) W_{0}+\sin \tau\left(J\left(T_{0}^{\prime}\right) W_{0}\right)
$$

while in the case $\xi_{1}^{0}=0, W_{0}$ is subject only to the condition

$$
2\left|\xi_{2}^{0}\right|=(\tau-\sin \tau)\left|W_{0}\right|^{2}
$$

and otherwise arbitrary. The length of the sub-Riemannian geodesics is

$$
\tau\left|W_{0}\right|
$$

Let $\mu(\tau)=(1-\cos \tau) /(\tau-\sin \tau)$. We consider the distribution of solutions in $[0, \infty)$ of the the equation

$$
\mu(\tau)=c
$$

where $c \in[0, \infty)$. The following lemma is elementary but paramountly important for the proof of Theorem 2.3.

LEMMA 2.2. For $0 \leqslant c<\infty$, we have

(1) if $c=0$, the solutions of (2.7) are $\tau=2 k \pi, k=1,2, \ldots$

(2) if $c>0$, then (2.7) has finitely many solutions and all of them are in $(0,+\infty)$. Moreover, (2.7) has only one solution in $(0,2 \pi)$ if and only if $f(2(\pi-\theta))<0$ where $\theta=\arctan (-(1 / c))$ and $f(\tau)=\sin (\tau+\theta)$ $-\tau \cos \theta-\sin \theta$. Finally, if $f(2(\pi-\theta)) \geqslant 0$, then the least solution must satisfy $\tau_{1} \in[\pi-\theta,(3 / 2) \pi-\theta]$.

Proof: (1) and the first part of (2) are trivial. Since

$$
\dot{\mu}(\tau)=\frac{4 \sin (\tau / 2) \cos (\tau / 2)((\tau / 2)-\tan (\tau / 2))}{(\tau-\sin \tau)^{2}}
$$

$\mu(\tau)$ is decreasing on $(0,2 \pi)$. From $\lim _{\tau \rightarrow 0+} \mu(\tau)=+\infty, \mu(2 \pi)=0$ we deduce that the equation (2.7) has exactly one solution in $[0,2 \pi]$. 
Let $c>0$ and $\theta=\arctan (-(1 / c)) \in(-(\pi / 2), 0)$, then (2.7) can be rewritten as

$$
\sin (\theta+\tau)=\tau \cos \theta+\sin \theta
$$

Let $f(\tau)=\sin (\tau+\theta)-\tau \cos \theta-\sin \theta$. We note that $f(2 \pi)=-2 \pi \cos \theta<0$ and $\dot{f}(\tau)=\cos (\tau+\theta)-\cos \theta>0$ whenever $\tau$ in $(2 \pi, 2(\pi-\theta))$. Thus by Rolle's Theorem (2.7) has exactly one solution in $(0,2 \pi)$ if and only if $f(2(\pi-\theta))<0$.

If $f(2(\pi-\theta))=-2((\pi-\theta) \cos \theta+\sin \theta) \geqslant 0$ then $f(\pi-\theta)=-((\pi-\theta) \cos \theta$ $+\sin \theta) \geqslant 0$. Let $g(\theta)=f((3 / 2) \pi-\theta)=-1-((3 / 2) \pi-\theta) \cos \theta-\sin \theta$. Since $\dot{g}(\theta)$ $=((3 / 2) \pi-\theta) \sin \theta<0$ whenever $\theta \in(-(\pi / 2), 0), g(\theta)<g(-(\pi / 2))=-2 \pi<0$. We get $\tau_{1} \in[\pi-\theta,(3 / 2) \pi-\theta]$ again by Rolle's Theorem.

Now we can prove one of the main results in this paper.

THEOREM 2.3. (Equations of shortest sub-Riemannian geodesics.) Let $p_{0}$ $=\left(\xi_{1}^{0}, \xi_{2}^{0}\right)(\neq 0)$ be a point in $G$ with the same notation $T_{0}^{\prime}$, as in Proposition 2.1 and let $\gamma(s)=\left(\xi_{1}(s), \xi_{2}(s)\right), s \in[0,1]$ be a shortest sub-Riemannian geodesic connecting 0 to $p_{0}$, then

(1) if $\xi_{2}^{0}=0$, the shortest sub-Riemannian geodesic is unique and its equation is (2.1). Its length is $\rho=\left|\xi_{1}^{0}\right|$.

(2) if $\xi_{2}^{0} \neq 0$ and $\xi_{1}^{0} \neq 0$, the shortest sub-Riemannian geodesic is also unique and its equation is

$$
\begin{aligned}
& \xi_{1}(s)=\left(\cos \left(s \tau_{1}\right)-1\right) W_{0}+\sin \left(s \tau_{1}\right)\left(J\left(T_{0}^{\prime}\right) W_{0}\right), \\
& \xi_{2}(s)=\frac{1}{2}\left(s \tau_{1}-\sin \left(s \tau_{1}\right)\right)\left|W_{0}\right|^{2} T_{0}^{\prime}
\end{aligned}
$$

where $\tau_{1} \in(0,2 \pi)$ is the least solution in $(0,+\infty)$ of equation (2.3) and $W_{0}$ is determined by (2.4) and (2.5) where $\tau$ is replaced by $\tau_{1}$. Its length is

$$
\rho=\sqrt{\frac{2\left|\xi_{2}^{0}\right| \tau_{1}^{2}}{\tau_{1}-\sin \tau_{1}}} .
$$

(3) if $\xi_{2}^{0} \neq 0$ and $\xi_{1}^{0}=0$, there are infinitely many shortest sub-Riemannian geodesics and their equations are

$$
\begin{aligned}
& \xi_{1}(s)=(\cos (2 \pi s)-1) W_{0}+\sin (2 \pi s)\left(J\left(T_{0}^{\prime}\right) W_{0}\right), \\
& \xi_{2}(s)=\frac{1}{2}(2 \pi s-\sin (2 \pi s))\left|W_{0}\right|^{2} T_{0}^{\prime}
\end{aligned}
$$

where $W_{0}$ is only subject to

$$
\left|\xi_{2}^{0}\right|=\pi\left|W_{0}\right|^{2}
$$

The length is 


$$
\rho=2 \sqrt{2 \pi\left|\xi_{2}^{0}\right|}
$$

Proof: Since every pair of points in $G$ can be joined by a shortest sub-Riemannian geodesic, (2.3) follows from Proposition 2.1.

If $\xi_{2}^{0} \neq 0$, from (2.5) we deduce that the length of a sub-Riemannian geodesic can be rewritten as

$$
\rho=\sqrt{\frac{2\left|\xi_{2}^{0}\right| \tau^{2}}{\tau-\sin \tau}} .
$$

Thus if $\xi_{1}^{0}=0$, it is obvious that $\tau$ corresponding to the shortest sub-Riemannian geodesic is $\tau_{1}=2 \pi$. Since there are infinitely many solutions to equation $(2.11),(2.3)$ follows.

If $\xi_{1}^{0} \neq 0$ and $\xi_{2}^{0} \neq 0$, we first note that for fixed $\tau(2.4)$ has only one solution in $V_{2}$. So in order to prove (2.3) it suffices to prove that the length of the sub-Riemannian geodesic corresponding to $\tau \in(2 \pi,+\infty)$ (if such $\tau$ exists) is strictly larger than the length of the sub-Riemannian geodesic corresponding to $\tau_{1} \in(0,2 \pi)$. When (2.3) has only one solution $\tau_{1} \in(0,2 \pi)$, it is obvious. If (2.3) has another solution $\tau_{2}$ except $\tau_{1}$, then by Lemma 2.2 $\tau_{1} \in[\pi-\theta,(3 / 2) \pi-\theta] \subset((3 / 2) \pi, 2 \pi)$ and hence $\sin \tau_{1}<0$. Let $\rho_{2}$ be the length of geodesic corresponding to $\tau_{2}$ determined by (2.13). In the case $\tau_{2} \in(2 \pi, 2(\pi-\theta)]$, since $\sin \tau_{2}>0$ and $\sin \tau_{1}<0$ we have

$$
\begin{aligned}
\rho_{2}^{2}-\rho_{1}^{2} & =2\left|\xi_{2}^{0}\right| \frac{\tau_{2} \tau_{1}\left(\tau_{2}-\tau_{1}\right)+\tau_{1}^{2} \sin \tau_{2}-\tau_{2}^{2} \sin \tau_{1}}{\left(\tau_{2}-\sin \tau_{2}\right)\left(\tau_{1}-\sin \tau_{1}\right)} \\
& >0
\end{aligned}
$$

In the case $\tau_{2} \in(2(\pi-\theta),+\infty)$, since

$$
\begin{aligned}
\tau_{2}-\tau_{1} & >2(\pi-\theta)-\left(\frac{3}{2} \pi-\theta\right) \\
& =\frac{\pi}{2}-\theta>1,
\end{aligned}
$$

we have

$$
\begin{aligned}
\rho_{2}^{2}-\rho_{1}^{2} & =2\left|\xi_{2}^{0}\right| \frac{\tau_{2} \tau_{1}\left(\tau_{2}-\tau_{1}\right)+\tau_{1}^{2} \sin \tau_{2}-\tau_{2}^{2} \sin \tau_{1}}{\left(\tau_{2}-\sin \tau_{2}\right)\left(\tau_{1}-\sin \tau_{1}\right)} \\
& \geqslant 2\left|\xi_{2}^{0}\right| \frac{\tau_{2} \tau_{1}\left(\tau_{2}-\tau_{1}\right)-\tau_{1}^{2}-\tau_{2}^{2} \sin \tau_{1}}{\left(\tau_{2}-\sin \tau_{2}\right)\left(\tau_{1}-\sin \tau_{1}\right)} \\
& >2\left|\xi_{2}^{0}\right| \frac{\tau_{1}\left(\tau_{2}-\tau_{1}\right)-\tau_{2}^{2} \sin \tau_{1}}{\left(\tau_{2}-\sin \tau_{2}\right)\left(\tau_{1}-\sin \tau_{1}\right)} \\
& >0
\end{aligned}
$$

Thus we have finished the proof.

For the Heisenberg group $\mathbb{H}^{n}$, since the map $J$ can be explicitly written as in (1.3), the following corollary follows immediately from Theorem 2.3. 
Corollary 2.4. Let $g_{0}=\left(x_{0}, y_{0}, t_{0}\right) \neq 0$ be a point in $\mathbb{H}^{\mathbf{n}}$. We have

(1) if $x_{0}^{2}+y_{0}^{2} \neq 0$, then there exists a unique shortest sub-Riemannian geodesic connecting 0 to $g_{0}$.

(2) otherwise, there exist infinitely many shortest sub-Riemannian geodesics connecting 0 to $g_{0}$.

Moreover, let $\gamma(s)=(x(s), y(s), t(s))(0 \leqslant s \leqslant 1)$ be any shortest sub-Riemannian geodesic connecting 0 to $g_{0}$, we have

$$
\begin{cases}x_{i}(s)=\frac{A_{i}(\cos (s \phi \rho)-1)+B_{i} \sin (s \phi \rho)}{\phi}, & i=1, \ldots, n \\ y_{i}(s)=\frac{B_{i}(\cos (s \phi \rho)-1)-A_{i} \sin (s \phi \rho)}{\phi}, & i=1, \ldots, n \\ t(s) & =\frac{s \phi \rho-\sin (s \phi \rho)}{2 \phi^{2}},\end{cases}
$$

where $\tau=\phi \rho \in[-2 \pi, 2 \pi]$ is the unique solution in $[-2 \pi, 2 \pi]$ of the equation

$$
\frac{1-\cos \tau}{\tau-\sin \tau}=\frac{\left|x_{0}\right|^{2}+\left|y_{0}\right|^{2}}{4 t_{0}}
$$

with

$$
\begin{cases}\tau=0 & \text { if } t_{0}=0 \\ |\tau|=2 \pi & \text { if }\left|x_{0}\right|^{2}+\left|y_{0}\right|^{2}=0 \\ \tau \in(0,2 \pi) & \text { if } t_{0}>0 \\ \tau \in(-2 \pi, 0) & \text { otherwise }\end{cases}
$$

$\rho=d_{c}\left(0, g_{0}\right)$ is the arc length of $\gamma$ determined by

$$
\begin{aligned}
& \rho=\sqrt{2 \frac{\tau^{2} t_{0}}{(\tau-\sin \tau)}}, \quad \text { if } t_{0} \neq 0, \\
& \rho=\sqrt{\left|x_{0}\right|^{2}+\left|y_{0}\right|^{2}}, \quad \text { if } t_{0}=0
\end{aligned}
$$

if $\left|x_{0}\right|^{2}+\left|y_{0}\right|^{2} \neq 0,\left\{A_{1}, \ldots, A_{n}, B_{1}, \ldots, B_{n}\right\}$ is subject to

$$
\begin{cases}\sum_{i=1}^{n}\left(A_{i}^{2}+B_{i}^{2}\right)=1, & \\ x_{0 i}=\frac{A_{i}(\cos (\phi \rho)-1)+B_{i} \sin (\phi \rho)}{\phi}, & i=1, \ldots, n \\ y_{0_{i}}=\frac{B_{i}(\cos (\phi \rho)-1)-A_{i} \sin (\phi \rho)}{\phi}, & i=1, \ldots, n\end{cases}
$$

if $\left|x_{0}\right|^{2}+\left|y_{0}\right|^{2}=0$, then $\left\{A_{1}, \ldots, A_{n}, B_{1}, \ldots, B_{n}\right\}$ is only subject to

$$
\sum_{i=1}^{n}\left(A_{i}^{2}+B_{i}^{2}\right)=1 \text {. }
$$


The following corollary, which follows immediately from Theorem 2.3 and the leftinvariance of the Carnot-Carathéodory metric $d_{c}$, will be used in Section 3.

COROLLARY 2.5. $\gamma$ is a sub-Riemannian geodesic connecting $p$ to $q$ if and only if $p^{-1} \gamma$ is a sub-Riemannian geodesic joining 0 and $p^{-1} q$. Moreover

(1) Let $\gamma(s)(s \in[0,+\infty))$ be a smooth arc-length parameterised curve emitting from 0 . Then $\gamma$ is a globally shortest geodesic (that is, $s_{2}-s_{1}$ $=d_{c}\left(\gamma\left(s_{2}\right), \gamma\left(s_{1}\right)\right)$ for any $s_{2}>s_{1}$ in $\left.[0,+\infty)\right)$ if and only if $\gamma$ ia a ray, that is, there exists an element $p_{0}=\left(\xi_{1}^{0}, 0\right) \in G^{*}$ such that $\left|\xi_{1}^{0}\right|=1$ and $\gamma(s)=s p_{0}(s \in[0,+\infty))$ where we abuse the notation $s g=\delta_{s} g$ when $p \in G^{*}$.

(2) Given two different points $p_{1}, p_{2} \in G$, then there are infinitely many shortest geodesics connecting them if and only if $p_{1}^{-1} p_{2} \in \exp \left(V_{2}\right)$, that is $\xi_{1}\left(p_{1}^{-1} \cdot p_{2}\right)=0$.

\section{Characterisation of the Sub-Riemannian Isometry Group}

In this section we give a full characterisation of the sub-Riemannian isometry group of a $H$-type group. Note that we shall not impose any smoothness conditions on an isometry.

We shall use $I S O(G)$ to denote the set of all sub-Riemannian isometries. Note that if $f$ is an sub-Riemannian isometry, then $g=f(0)^{-1} f$ is an sub-Riemannian isometry preserving the unit.

\section{LEMMA 3.1.}

(1) Let $p_{1}, p_{2}$ be two different points in $G$ and $f$ be a sub-Riemannian isometry. Then $\gamma$ is a shortest geodesic connecting $p_{1}$ to $p_{2}$ if and only if $f(\gamma)$ is a shortest geodesic connecting $f\left(p_{1}\right)$ to $f\left(p_{2}\right)$. In particular, if $f$ is an isometry fixing the unit, then $\gamma$ is a ray emitting from 0 if and only if $f(\gamma)$ is a ray from 0 .

(2) If $p \in \exp \left(V_{2}\right)$ and $f$ is an isometry preserving the unit, then $f(p)$ is also in $\exp \left(V_{2}\right)$.

ProOF: Without restriction we assume that all shortest sub-Riemannian geodesics are parameterised by arc length. Let $\gamma(s)\left(s \in\left[0, d_{c}\left(p_{1}, p_{2}\right)\right]\right)$ be a shortest subRiemannian geodesic joining $p_{1}$ to $p_{2}$. By definition

$$
s_{2}-s_{1}=d_{c}\left(\gamma\left(s_{1}\right), \gamma\left(s_{2}\right)\right)=d_{c}\left(f\left(\gamma\left(s_{1}\right)\right), f\left(\gamma\left(s_{2}\right)\right)\right)
$$

for any $s_{2}>s_{1}$ in $\left[0, d_{c}\left(p_{1}, p_{2}\right)\right]$. So it follows from Pansu's Theorem on differentiability of Lipschitz functions defined on Carnot groups ([22]) that $f(\gamma)$ is horizontal. Thus (3.1) means that $f(\gamma)$ is a shortest geodesic connecting $f\left(p_{1}\right)$ to $f\left(p_{2}\right)$. If $f$ is an isometry 
preserving the unit and $\gamma$ is a ray starting from 0 , then $f(\gamma)$ is a globally shortest geodesic. By Corollary $2.5, f(\gamma)$ is a ray from 0 . Since the inverse of an isometry is also an isometry, we proved (3.1).

Because of $p \in \exp \left(V_{2}\right)$ there are infinitely many geodesics connecting 0 to $p$ by Corollary 2.5. Let $\gamma$ be any such geodesic. By $(3.1), f(\gamma)$ is a shortest geodesic connecting 0 to $f(p)$. Thus there are infinitely many shortest geodesics joining 0 and $f(p)$. So $f(p)$ is in $\exp \left(V_{2}\right)$ again by Corollary 2.5 .

Proposition 3.2. Any sub-Riemannian isometry with $f(0)=0$ can be written as

$$
f(p)=\left(f_{1}\left(\xi_{1}(p)\right), f_{2}\left(\xi_{2}(p)\right)\right)
$$

for $p=\left(\xi_{1}(p), \xi_{2}(p)\right)$, where $f_{1} \in \mathcal{O}\left(V_{1}\right), f_{2} \in \mathcal{O}\left(V_{2}\right)$ and $\mathcal{O}\left(V_{i}\right)$ is the orthogonal group of $V_{i}, i=1,2$.

Proof: Let $f$ be a sub-Riemannian isometry with $f(0)=0$. By Lemma 3.1, $f(p)$ $\in G^{*}$ for $p \in G^{*}$ and $f\left(p^{\prime}\right) \in \exp \left(V_{2}\right)$ for $p^{\prime} \in \exp \left(V_{2}\right)$. Now let $p$ be any point in $G$ and $p^{\prime}$ be any point in $G^{*}$. Let $\gamma(s)=p$.sp be a ray joining $p$ and $p^{\prime}$. Since $f(\gamma(s))$ is a ray joining $f(p) \in G$ and $f\left(p^{\prime}\right) \in G^{*}$, there exist $\widetilde{p} \in G$ and $\widetilde{p}$ such that $f(\gamma(s))=\tilde{p} \cdot s \tilde{p}$. We deduce that

$$
f\left(p p^{\prime}\right)=f(p) f\left(p^{\prime}\right) \text { for any } p \in G, p^{\prime} \in G^{*} .
$$

For $f$ we define two functions $f_{1}$ and $f_{2}$ on $V_{1}$ and $V_{2}$ respectively:

$$
f_{1}\left(\xi_{1}\right):=\xi_{1}\left(f\left(\left(\xi_{1}, 0\right)\right)\right), \quad f_{2}\left(\xi_{2}\right):=\xi_{2}\left(f\left(\left(0, \xi_{2}\right)\right)\right) .
$$

Then by (3.2), for any $p^{\prime}=\left(\xi_{1}^{\prime}, \xi_{2}^{\prime}\right)$ we have

$$
\begin{aligned}
f\left(\left(\xi_{1}^{\prime}, \xi_{2}^{\prime}\right)\right) & =f\left(\left(0, \xi_{2}^{\prime}\right)\left(\xi_{1}^{\prime}, 0\right)\right)=f\left(\left(0, \xi_{2}^{\prime}\right)\right) f\left(\left(\xi_{1}^{\prime}, 0\right)\right) \\
& =\left(0, \xi_{2}\left(f\left(\left(0, \xi_{2}^{\prime}\right)\right)\right)\right)\left(\xi_{1}\left(f\left(\left(\xi_{1}^{\prime}, 0\right)\right)\right), 0\right) \\
& =\left(\xi_{1}\left(f\left(\left(\xi_{1}^{\prime}, 0\right)\right)\right), \xi_{2}\left(f\left(\left(0, \xi_{2}^{\prime}\right)\right)\right)\right) \\
& =\left(f_{1}\left(\xi_{1}^{\prime}\right), f_{2}\left(\xi_{2}^{\prime}\right)\right) .
\end{aligned}
$$

Thus for $p=\left(\xi_{1}(p), \xi_{2}(p)\right)$, we can write

$$
f(p)=\left(f_{1}\left(\xi_{1}(p)\right), f_{2}\left(\xi_{2}(p)\right)\right) .
$$

Let $p=\left(\xi_{1}, 0\right)$ be a point in $G^{*}$. On one hand by (2.3) in Theorem 2.3, we have $\left|\xi_{1}\right|=d_{c}\left(\left(\xi_{1}, 0\right), 0\right)=d_{c}(p, 0)=d_{c}(f(p), 0)=d_{c}\left(\left(f_{1}\left(\xi_{1}\right), 0\right), 0\right)=\left|f_{1}\left(\xi_{1}\right)\right|$. One the other hand by (3.2), $f_{1}$ is a linear map from $V_{1}$ to $V_{1}$. Thus $f_{1}$ is an orthogonal transformation in $V_{1}$. 
Let $p_{i}=\left(0, \xi_{2}^{i}\right), i=1,2$ be two points in $\exp \left(V_{2}\right)$. By (2.3) in Theorem 2.3 we have

$$
\begin{aligned}
d_{c}\left(f\left(p_{1}\right), f\left(p_{2}\right)\right) & =d_{c}\left(\left(0, f_{2}\left(\xi_{2}^{1}\right)\right),\left(0, f_{2}\left(\xi_{2}^{2}\right)\right)\right) \\
& =d_{c}\left(0,\left(0, f_{2}\left(\xi_{2}^{1}\right)\right)^{-1} \cdot\left(0, f_{2}\left(\xi_{2}^{2}\right)\right)\right) \\
& =d_{c}\left(0,\left(0, f_{2}\left(\xi_{2}^{2}\right)-f_{2}\left(\xi_{2}^{1}\right)\right)\right) \\
& =2 \sqrt{2 \pi\left|f_{2}\left(\xi_{2}^{2}\right)-f_{2}\left(\xi_{2}^{1}\right)\right|}
\end{aligned}
$$

and

$$
\begin{aligned}
d_{c}\left(p_{1}, p_{2}\right) & =d_{c}\left(0, p_{1}^{-1} \cdot p_{2}\right) \\
& =d_{c}\left(0,\left(0, \xi_{2}^{2}-\xi_{2}^{1}\right)\right) \\
& =2 \sqrt{2 \pi\left|\xi_{2}^{2}-\xi_{2}^{1}\right|} .
\end{aligned}
$$

Since $d_{c}\left(f\left(p_{1}\right), f\left(p_{2}\right)\right)=d_{c}\left(p_{1}, p_{2}\right)$, we get $\left|f_{2}\left(\xi_{2}^{2}\right)-f_{2}\left(\xi_{2}^{1}\right)\right|=\left|\xi_{2}^{2}-\xi_{2}^{1}\right|$. Thus $f_{2}$ is an isometry in $V_{2}$. By [10, Section 2.3], $f_{2}$ is an orthogonal transformation.

Proposition 3.3. Any isometry with $f(0)=0$ satisfies that

$$
f\left(\delta_{s} p\right)=\delta_{s} f(p) \text { and } f\left(p p^{\prime}\right)=f(p) f\left(p^{\prime}\right)
$$

for any $s>0$ and $p, p^{\prime} \in G$.

ProOF: In fact by (3.2), (3.3) and Proposition 3.2, we have

$$
f\left(\delta_{s} p\right)=f\left(\left(s \xi_{1}, s^{2} \xi_{2}\right)\right)=\left(f_{1}\left(s \xi_{1}\right), f_{2}\left(s^{2} \xi_{2}\right)\right)=\left(s f_{1}\left(\xi_{1}\right), s^{2} f_{2}\left(\xi_{2}\right)\right)=\delta_{s} f(p)
$$

and

$$
\begin{aligned}
f\left(p p^{\prime}\right) & =f\left(\left(\xi_{1}, \xi_{2}\right)\left(\xi_{1}^{\prime}, \xi_{2}^{\prime}\right)\right)=f\left(\left(\xi_{1}, \xi_{2}\right)\left(0, \xi_{2}^{\prime}\right)\left(\xi_{1}^{\prime}, 0\right)\right) \\
& =f\left(\left(\xi_{1}, \xi_{2}+\xi_{2}^{\prime}\right)\right) f\left(\left(\xi_{1}^{\prime}, 0\right)\right)=\left(f_{1}\left(\xi_{1}\right), f_{2}\left(\xi_{2}+\xi_{2}^{\prime}\right)\right)\left(f_{1}\left(\xi_{1}^{\prime}\right), 0\right) \\
& =\left(f_{1}\left(\xi_{1}\right), f_{2}\left(\xi_{2}\right)+f_{2}\left(\xi_{2}^{\prime}\right)\right)\left(f_{1}\left(\xi_{1}^{\prime}\right), 0\right)=\left(f_{1}\left(\xi_{1}\right), f_{2}\left(\xi_{2}\right)\right)\left(0, f_{2}\left(\xi_{2}^{\prime}\right)\right)\left(f_{1}\left(\xi_{1}^{\prime}\right), 0\right) \\
& =f(p)\left(f_{1}\left(\xi_{1}^{\prime}\right), f_{2}\left(\xi_{2}^{\prime}\right)\right)=f(p) f\left(p^{\prime}\right)
\end{aligned}
$$

for any $p=\left(\xi_{1}, \xi_{2}\right), p^{\prime}=\left(\xi_{1}^{\prime}, \xi_{2}^{\prime}\right)$ in $G$ and any $s>0$.

Now we can prove another of the main results in this paper.

THEOREM 3.4. Let $f$ be a map from $G$ to $G$ with $f(0)=0$. Then $f$ is an isometry if and only if

$$
f(p)=\left(f_{1}\left(\xi_{1}\right), f_{2}\left(\xi_{2}\right)\right), f_{1} \in \mathcal{O}\left(V_{1}\right), f_{2} \in \mathcal{O}\left(V_{2}\right)
$$

and

$$
J\left(f_{2}\left(\xi_{2}\right)\right)\left(f_{1}\left(\xi_{1}\right)\right)=f_{1}\left(J\left(\xi_{2}\right)\left(\xi_{1}\right)\right)
$$

for any $p=\left(\xi_{1}, \xi_{2}\right) \in G$. 
Proof: Let's first show that for $f$ satisfying (3.4) with $f(0)=0,(3.5)$ is equivalent to the fact that $f$ is a group homomorphism. To this aim, let $p=\left(\xi_{1}, \xi_{2}\right), p^{\prime}=\left(\xi_{1}^{\prime}, \xi_{2}^{\prime}\right)$ and let $f$ be satisfying (3.4) for $f_{1} \in \mathcal{O}\left(V_{1}\right)$ and $f_{2} \in \mathcal{O}\left(V_{2}\right)$. By the Baker-HausdorffCampbell formula

$$
p p^{\prime}=\left(\xi_{1}+\xi_{1}^{\prime}, \xi_{2}+\xi_{2}^{\prime}+\frac{1}{2}\left[\xi_{1}, \xi_{1}^{\prime}\right]\right)
$$

we have

$$
f\left(p p^{\prime}\right)=\left(f_{1}\left(\xi_{1}\right)+f_{1}\left(\xi_{1}^{\prime}\right), f_{2}\left(\xi_{2}\right)+f_{2}\left(\xi_{2}^{\prime}\right)+\frac{1}{2} f_{2}\left(\left[\xi_{1}, \xi_{1}^{\prime}\right]\right)\right)
$$

and

$$
f(p) f\left(p^{\prime}\right)=\left(f_{1}\left(\xi_{1}\right)+f_{1}\left(\xi_{1}^{\prime}\right), f_{2}\left(\xi_{2}\right)+f_{2}\left(\xi_{2}^{\prime}\right)+\frac{1}{2}\left[f_{1}\left(\xi_{1}\right), f_{2}\left(\xi_{1}^{\prime}\right)\right]\right) .
$$

Thus $f$ is a group homomorphism if and only if

$$
\left[f_{1}\left(\xi_{1}\right), f_{1}\left(\xi_{1}^{\prime}\right)\right]=f_{2}\left(\left[\xi_{1}, \xi_{1}^{\prime}\right]\right) .
$$

If $f$ satisfies (3.5), recalling (1.1) we obtain

$$
\begin{aligned}
\left\langle\xi_{2}, f_{2}^{-1}\left[f_{1}\left(\xi_{1}\right), f_{1}\left(\xi_{1}^{\prime}\right)\right]\right\rangle & \stackrel{(3.4)}{=}\left\langle f_{2}\left(\xi_{2}\right),\left[f_{1}\left(\xi_{1}\right), f_{1}\left(\xi_{1}^{\prime}\right)\right]\right\rangle \stackrel{(1.1)}{=}\left\langle J\left(f_{2}\left(\xi_{2}\right)\left(f_{1}\left(\xi_{1}\right)\right)\right), f_{1}\left(\xi_{1}^{\prime}\right)\right\rangle \\
& \stackrel{(3.5)}{=}\left\langle f_{1}\left(J\left(\xi_{2}\right)\left(\xi_{1}\right)\right), f_{1}\left(\xi_{1}^{\prime}\right)\right\rangle \stackrel{(3.4)}{=}\left\langle J\left(\xi_{2}\right)\left(\xi_{1}\right), \xi_{1}^{\prime}\right\rangle \\
& \stackrel{(1.1)}{=}\left\langle\xi_{2},\left[\xi_{1}, \xi_{1}^{\prime}\right]\right\rangle .
\end{aligned}
$$

So (3.6) holds. The proof of the converse can be done similarly.

If $f$ is a sub-Riemannian isometry, then by Proposition 3.2 and Proposition 3.3 and the last statement, (3.4) and (3.5) hold.

If (3.4) and (3.5) hold, then the fact that $f$ is a group homomorphism implies that $f$ transforms horizontal curves into horizontal curves and (3.4) implies that it preserves their length. Of course, this implies that $f$ is an isometry.

Kaplan in [12] proved that a map $f$ fixing the unit is an isometry with respect to the standard Riemannian metric $\langle\cdot, \cdot\rangle$ if and only if (3.4) and (3.5) hold. The set of all maps satisfying (3.4) and (3.5) is denoted by $A(G)$, also called the automorphism group of $G$.

THEOREM 3.5. The sub-Riemannian isometry group $I S O(G)$ coincides with the isometry group with respect to the standard Riemannian metric $\langle\cdot, \cdot\rangle$. That is, $I S O(G)$ is the semidirect product $A(G) \times G$ (with $G$ acting by left translation).

In the Heisenberg group $\mathbb{H}^{n}$, the set $A(G)$ can be more explicitly described due to the fact that $V_{1} \simeq \mathbb{R}^{2 n}$ can be endowed with a symplectic structure and $V_{2}$ is of one dimension and so the map $J$ can be explicitly written out (see (1.3)).

COROLlary 3.6. 
(1) In $\mathbb{H}^{n}$, the unit component $A_{0}\left(\mathbb{H}^{n}\right)$ of the automorphism group $A\left(\mathbb{H}^{n}\right)$ can be identified with the Unitary group $\mathcal{U}(n)$ in the following sense: let $f \in A_{0}\left(\mathbb{H}^{n}\right)$, then

$$
f=\left[\begin{array}{ll}
U & 0 \\
0 & 1
\end{array}\right]
$$

where $U \in \mathcal{U}(n)$ and $0 \in \mathbb{R}^{2 n}$.

(2) Another component $A_{1}\left(\mathbb{H}^{n}\right)$ of $A\left(\mathbb{H}^{n}\right)$ is the product of $A_{0}\left(\mathbb{H}^{n}\right)$ by the matrix

$$
\left[\begin{array}{ccc}
E & 0 & 0 \\
0 & -E & 0 \\
0 & 0 & -1
\end{array}\right]
$$

where $E$ is the unit matrix of $n \times n$.

Proof: From the group law (1.2) we easily deduce that

$$
\left[z, z^{\prime}\right]=\frac{1}{2} \sum_{i=1}^{n}\left(x_{i}^{\prime} y_{i}-x_{i} y_{i}^{\prime}\right)=\frac{1}{2} \omega\left(z, z^{\prime}\right)
$$

for $z=(x, y), z^{\prime}=\left(x^{\prime}, y^{\prime}\right)$ in $\mathbb{R}^{2 n}$. In $(3.9), \omega\left(z, z^{\prime}\right)$ denotes the standard symplectic form in $\mathbb{R}^{2 n}$.

Let $f \in A\left(\mathbb{H}^{n}\right)$. Then by (3.4), $f$ can be written as $f(p)=\left(f_{1}(z), f_{2}(t)\right)$ for $p=(z, t)=(x, y, t), z=(x, y)$ where $f_{1} \in \mathcal{O}\left(\mathbb{R}^{2 n}\right)$ and $f_{2}(t)=t$ or $f_{2}(t)=-t$ for any $t \in \mathbb{R}$. By (3.5), (3.6) and (3.9) we have if $f_{2}(t)=t$ for any $t \in \mathbb{R}$, then

$$
\omega\left(f_{1}(z), f_{1}\left(z^{\prime}\right)\right)=\omega\left(z, z^{\prime}\right)
$$

and if $f_{2}(t)=-t$ for any $t \in \mathbb{R}$, then

$$
\omega\left(f_{1}(z), f_{1}\left(z^{\prime}\right)\right)=-\omega\left(z, z^{\prime}\right) .
$$

Note that (3.10) means that $f_{1}$ is a symplectic transformation and (3.11) means that $f_{1}$ can be seen as the composition of a symplectic transformation with a map determined by the matrix (3.8). We use $\mathcal{S} p(n, \mathbb{R})$ to denote the symplectic group in $\mathbb{R}^{2 n}$.

It is easy to verify that if $f \in A_{0}\left(\mathbb{H}^{n}\right)$ (or $f \in A_{1}\left(\mathbb{H}^{n}\right)$ ), then $f_{2}(t)=t$ (or $-t$ ) for any $t \in \mathbb{R}$.

Thus we infer that for $f \in A_{0}\left(\mathbb{H}^{n}\right), f((z, t))=\left(f_{1}(z), t\right)$ where $f_{1} \in O\left(\mathbb{R}^{2 n}\right) \cap \mathcal{S} p(n, \mathbb{R})$ $=\mathcal{U}(n)$ (see for example [1]). This completes the proof of (1). (2) follows from (1) and the above argument.

REMARK. The full characterisation of the sub-Riemannian isometry group of the Heisenberg group may be useful in finding out the exact isoperimetric set in the Heisenberg 
group ([17]). In Euclidean case, one can use symmetrisation techniques to prove the isoperimetric set is spherical. This is due to the fact that the isometry group of $\mathbb{R}^{n}$ is large enough to give information of any direction when one tries to deform a set using an isometry. But in the case of the Heisenberg group it is still an open problem whether there are similar symmetrisation result. For this topic we refer to $[17,18]$.

\section{REFERENCES}

[1] B. Aebischer, M. Borer, M. Kälin, C. Leuenberger and H.M. Reimann, Symplectic geometry, Progress in Mathematics 124 (Birkhäuser, Basel, 1992).

[2] L. Ambrosio and S. Rigot, 'Optimal mass transportation in the Heisenberg group', $J$. Funct. Anal. (to appear).

[3] R. Beals, B. Gaveau and P.C. Greiner, 'Hamilton-Jacobi theory and heat kernel on Heisenberg groups', J. Math. Pures Appl. 79 (2000), 633-689.

[4] A. Bellaiche, The tangent spaces in sub-Riemannian geometry, Progress in Mathematics 144 (Birkhäuser, Basel, 1996).

[5] J. Berndt, F. Tricerri and L. Vanhecke, Generalized Heisenberg groups and Damek-Ricci harmonic spaces, Lecture Notes in Math. 1598 (Springer-Verlag, Berlin, Heidelberg, New York 1994).

[6] W.L. Chow, 'über Systeme non linearen partiellen Differentialgleichungen erster Ordung', Math. Ann. 117 (1939), 98-105.

[7] G.B. Folland and E.M. Stein, Hardy spaces on homogeneous groups (Princeton Univ. Press, Princeton, N.J., 1982).

[8] M. Gromov, Metric structures for Riemannian and non-Riemannian spaces, Progress in Mathematics 152 (Birkhäuser, Boston, 1999).

[9] M. Gromov, Carnot-Carathéodory spaces seen from within, Progress in Mathematics 144 (Birkhäuser, Basel, 1996).

[10] J.E. Hutchinson, 'Fractals and self similarity', Indiana. Univ. Math. J. 30 (1981), 713-747.

[11] A. Kaplan, 'Fundamental solutions for a class of hypoelliptic PDE generated by composition of quadratic forms', Trans. Amer. Math. Soc. 258 (1980), 147-153.

[12] A. Kaplan, 'Riemannian nilmanifolds attached to Clifford modules', Geom. Dedicata, 11 (1981), 127-136.

[13] A. Korányi, 'Geometric properties of Heisenberg-type groups', Adv. in Math. 56 (1985), 28-38.

[14] A. Korányi and H.M. Reimann, 'Quasiconformal mappings on the Heisenberg group', Invent. Math. 80 (1985), 309-338.

[15] A. Koranyi, Geometric aspects of analysis on the Heisenberg group, Topics in modern harmonic analysis, Vol. I, II (Turin/Milan, 1982), 209C258 (Ist. Naz. Alta Mat. Francesco Severi, Rome, 1983).

[16] A. Korányi and H.M. Reimann, 'Foundations for the theory of quasiconformal mappings on the Heisenberg group', Adv. Math. 111 (1995), 1-87.

[17] G.P. Leonardi and S. Masnou, 'On the isoperimetric problems in the Heisenberg group $\mathbb{H}^{n}$, (preprint 2002) available from http://cvgmt.sns.it. 
[18] G.P. Leonardi and S. Rigot, 'Isoperimetric sets on Carnot groups', Houston J. Math. 29 (2003), 609-637.

[19] W.S. Liu and H. Sussman, 'Shortest paths for sub-Riemannian metrics on rank-two distributions', Mem. Amer. Math. Soc. 118 (1995).

[20] R. Monti, 'Some properties of Carnot-Caratheodory balls in the Heisenberg group', Atti. Accad. Naz. Lincei. Cl. Sci. Fis. Mat. Natur. Ren. Lincei (9) Mat. Appl. 11 (2000), 155-167.

[21] R. Montgomery, A tour of sub-Riemannian geometry, their geodesics and applications, Mathematical Surveys and Monographs 91 (American Mathematical Society, Providence R.I., 2002).

[22] P. Pansu, 'Métriques de CC et quasiisométries des espaces symétriques de rang un', $A n n$. of Math. 119 (1989), 1-60.

[23] E.M. Stein, Harmonic analysis (Princeton University press, Princeton N.J., 1993).

[24] K.H. Tan, 'Sobolev classes and horizontal energy minimizers between CarnotCarathéodory spaces', Commun. Contemp. Math. 6 (2004), 1-33.

Department of Applied Mathematics

Science School

Nanjing University of Science and Technology

210094, Nanjing

Peoples Republic China

e-mail: tankanghai2000@yahoo.com.cn

yangxp@mail.njust.edu.cn 